\title{
How Students Communicate Mathematical Ideas: An Examination of Multimodal Writing Using Digital Technologies
}

\author{
Barbara Freeman \\ University of California at Berkeley, United States \\ Kristina N. Higgins \\ Tarleton State University, United States \\ Mark Horney \\ University of Oregon, United States
}

\begin{abstract}
This article reports the findings of a study designed to examine the influence of multimodal writing on the communication of mathematical ideas. Elementary school students (ages 8-13) were required to write mathematics notes using two digital writing technologies, a personal digital notepad and a social mathematics blog, in the context of a formal intervention. Fortytwo students participated, across three schools. The study showed that when students wrote notes that could be assessed for correctness, their answers were predominately right, indicating that mathematical sense-making was taking place. It also showed that the digital notepad and blog were used differently and that the type of technology influences the writing content. Moreover, students' mathematical writing were understandable by their peers and students collaboratively explored solutions. Younger students were more likely to draw pictures to represent their ideas than older students. These findings show that writing can help students acquire mathematical understanding, and suggest that multimodal writing may help them surmount difficulties often associated with learning math. Although this research demonstrated that writing can help teachers gain an awareness of their students' mathematical understanding, it also revealed that writing environments need to be monitored and students require close guidance to bring about systematic improvement.
\end{abstract}

Keywords: Mathematics education; Instructional technology; Elementary education; Digital writing technologies; Blogging; Mathematics journal

\section{Introduction}

A common assumption in education is that students should be able to express themselves in writing. In recent years, this expectation has expanded from its origins in the Language Arts curriculum and is ensconced in all the content areas, including mathematics. This requirement is now firmly established in curriculum standards reflected in statements such as these, drawn from the US national Common Core State Standards Initiative (US NGA \& US CCSSO, 2010): 
"Mathematically proficient students can explain correspondences between equations, verbal descriptions, tables, and graphs or draw diagrams of important features and relationships, graph data, and search for regularity or trends" (p. 6).

"They justify their conclusions, communicate them to others, and respond to the arguments of others" (p. 7).

"Mathematically proficient students try to communicate precisely to others" (p. 7).

Although these writing standards are well established and digital technologies that enable mathematical writing continually improve, the researchers' observations across multiple schools in many settings led them to question whether this writing requirement was perhaps less practiced by students or expected by teachers than might be hoped. The researchers thus designed a rigorous study to formally test the relationships between writing, mathematics, and digital technologies in order to better understand how students communicate their mathematical ideas. This was tested through two related research studies of elementary school students (ages 8-13), who were required to use two different digital writing technologies: a personal digital notepad and a social mathematics blog. A formal protocol was used for the intervention, which was conducted in the context of an online supplemental mathematics curriculum.

This article begins with a discussion of the importance of writing in mathematics and a survey of the relevant literature and research, especially that which pertains to mathematical communication using digital technologies. This is followed by a presentation of the study methodology and results. The paper concludes with a discussion of the findings and recommendations for future action.

\section{Writing in Mathematics}

Lee (2010) details how writing in the context of mathematics assists in learning and retaining mathematical concepts, arguing that "...being able to write clearly is as important a mathematical skill as being able to solve equations" (p. 1). Essentially, writing in mathematics-like writing in all non-literary matters -is about communicating ideas with clarity and an appropriate level of detail to make these ideas understandable and traceable. The relationship between writing in mathematics and mathematical thinking, however, is intertwined and complex. Writing represents thought, which demands conscious awareness and intention on the part of the writer (Vygotsky, 1986). A student's writing renders his or her thinking more visible. When a student is able to express and explain her reasoning and justify her thought processes and solutions correctly in writing, it shows her command of the concept (Baxter, Woodward, \& Olson, 2005; Gould, 2013). As a student moves through the levels from an absence of understanding or, perhaps worse, misunderstanding, through to mastery, she is aided by the process of writing and rewriting, and questioning and answering, wherein her conceptual understanding is embellished and embedded. Successive iterations of writing and revision (contemplation) lay down deeper layers of understanding through deliberation and analysis (Ong, 1982).

When a student explains in writing the logic of her answer for her own purposes (e.g., in a journal), the explanation needs to be somewhat logically coherent (even if based on misconceptions) in order 
that she can follow her processes at a later date (Devlin, 2012). When a student, however, shares her logic or analysis with someone else through writing, it must be written with precision and clarity in order to render its meaning understandable to the intended reader. Hence, not only must the mathematical reasoning be logical and sound, but the communication must also be coherent and sound. This underscores the key point that mathematical reasoning and communication of that mathematical reasoning are symbiotic but they are not one in the same thing.

An interdependent connection also exists between writing and technology. As Haas (2013) argues "different writing technologies can support very different mental processes" (p. xiv). This is important because the technologies used in US classrooms-from kindergarten to high school (K12)-are changing. Increasingly, students work on their class assignments and homework using digital technologies, such as Google documents, social blogs, and so forth. The writing that is produced using these technologies is easily sharable, updatable, and portable, as well as collaborative and social in nature. Furthermore, these technologies change the way writing is recorded, in that it becomes more organic: a blog, for example, becomes the product of social interaction. Technologies such as these enable students to easily share their work with broader audiences (Scardamalia \& Bereiter, 1994) and to do so in real time. This, in turn, places greater importance on the interdependent functions of mathematical thinking and communication of that mathematical reasoning.

A plethora of literature describes and substantiates the critical relationship between writing and mathematics (Bicer, Capraro, \& Capraro, 2013; Burns, 1995, 2004). Scholars have written extensively about writing and technology throughout the decades (Haas, 2013; Havelock, 1986; Ong, 1982; Scardamalia \& Bereiter, 1994). Furthermore, several meta-analyses and best evidence syntheses review a vast body of literature that details the connection between mathematics and technology (Huscroft-D’Angelo, Higgins, \& Crawford, 2016; Li \& Ma, 2010; Slavin \& Lake, 2008; Slavin, Lake, and Groff, 2009). Yet, there is a paucity of research around the interrelationship amongst these three key components: writing, mathematics, and digital technologies. We know that the writing process is affected by changing technologies, as this is largely observable from looking at students' changing study and learning practices (e.g., printed private notebooks to on-demand digital tutors; Haas, 2013). What is under-researched is the way that these three intertwined components influence students' communication of their mathematical ideas.

\section{Multimodal Writing in Formal and Informal Contexts}

Foremost, when contemplating writing in mathematics, we must recognize it to be multimodal in nature (Schleppegrell, 2010). Writing typically takes many forms, including language (written text and recordings of verbal communication), numbers, formulas and mathematical symbols, and visual representations (graphs, diagrams, charts, models, pictures, maps, flow charts). Multimodal writing is needed in both formal and less formal contexts.

Formal writing in mathematics is a precise language that requires accuracy in its expression, especially at higher levels of mathematics study (Devlin, 2012), though it also constitutes a large part of K-12 education: in the classroom, in textbooks, and on assessments. The language of mathematics contains mathematical statements (hypotheses, conjectures, axioms, and theorems), 
linguistic forms and properties, grammar (connectors, combinators), and symbols. This language is often information-dense and abstract (Schleppegrell, 2007). It is also vastly different than language used in social conversation (Cummins, 1979), as is the vocabulary of mathematics with mathematical meanings being much more exact and nuanced than their ordinary definitions (Freeman, 2012, 2013). It is not uncommon for students to give up learning a mathematical concept because they missed a step in their teacher's explanation or in the textbook (mathematics software program, etc.) or, conversely, their teachers or textbooks left gaps in their explanation (Ash, n.d.).

Effective communication-requiring more informal styles of writing that use everyday language and visual representation-is especially vital given that independent study is increasingly part of formal $\mathrm{K}-12$ schooling and the principal means of acquiring new skills and competencies throughout one's life, be it for career retraining or advancement, or for lifelong learning. When writing in a Google doc or on a social blog, for example, students are writing in an environment in which the expectation is that they will receive comments and immediate feedback from their teachers and possibly their peers. Thus, such writing is generally accepted as dialogical, and output is often treated as working drafts (Kenney, Hancewicz, Heuer, Metsisto, \& Tuttle, 2005).

\section{Writing and the US Common Core}

The US National Council for Teachers of Mathematics (NCTM) argue that students need to develop mathematical ways of thinking in STEM domains and in the real world (US NCTM, 2014). The US Common Core State Standards in Mathematics (CCSSM) similarly require students to develop mathematical thinking skills, which are distinct from the CCSSM content standards yet are intended to cut across all grade levels of the content standards (US NGA \& US CCSSO, 2010). There are eight mathematical practices that we abbreviate as MP1, MP2, MP3, etc.

Writing is integral to "construct viable arguments and critique the reasoning of others" (MP3). Students are not only supposed to derive answers to mathematics problems, they are also expected to know how and justify why they arrived at their answers, and communicate this understanding to others. This practice is considered fundamental to the development of mathematical proficiency and literacy.

Writing is also a constituent part of the other CCSSM practice standards, and substantiates the evidence-based link between students' writing and their learning (US National Institute for Literacy, 2007). For example, one of the practice standards requires students to "make sense of problems and persevere in solving them" (MP1). Writing provides students with an opportunity to work through a solution to reach conceptual understanding of increasingly complex problems and to reflect on their solution to the problem, considering whether it makes sense (Devlin, 2012; Pugalee, 2004). This means that students need to find a way in to the solution, which often requires making connections between new and prior knowledge (Bicer et al., 2013).

Other practice standards require students to "reason abstractly and quantitatively" (MP2), to "model with mathematics" (MP4), and to "attend to precision" (MP6). When students write in mathematics they are compelled to think mathematically, that is logically and analytically (Devlin, 2012). Mathematical proficiency requires students to represent quantitative relationships abstractly 
and to manipulate quantities within contextualized problems. This, in turn, requires students to use multimodal writing both symbolically (e.g., express logic using mathematical equations) and through visual representation (e.g., show mathematical relationships through graphs, diagrams). Alongside these key practice standards is a standard that demands students "look for and making use of structure" (MP7). Through deliberate analysis, writing has the potential to help students to compare, contrast, analyze, and synthesize information (Emig, 1977), which will help them achieve these competencies.

Akin to Common Core content standards, learning objectives for Common Core mathematical practices are developmental and progress across the grades. For students in elementary school, writing may comprise providing logical explanations for their mathematical solutions; whereas for secondary students (and beyond), writing may include inferring coherent arguments from established proofs and established axioms (Oregon Department of Education, 2012).

\section{Learning and Communicating Mathematical Ideas through Writing}

The idea that writing in mathematics is powerful is not a new discovery. Bruner (1968) observed that writing and mathematics are "devices for ordering thoughts about things and thoughts about thoughts" (p. 112). Learning to write in the context of mathematics allows students to pose questions and explore ideas (for example, "I don't get why the angles add up to 180 degrees"). Multimodal writing provides ways for students to gain insight into abstract concepts through drawing and other means of creative expression and experimentation. It also enables students to communicate their ideas to others, including their teachers and peers. Thus, writing can provide teachers with a window into their students' minds and what they are thinking (Ball, 1994; Bagley \& Gallenberger, 1992). Teachers can read what students are writing and students' writing is often reflective of their thinking, both their understanding and their misconceptions (Ashlock, 2006). It therefore provides a means of formative assessment (Burns, 2004), which enables the teacher to identify the point at which the student's incomprehension led to a wrong solution (Kenney et al., 2005; Wiliam, 2011). This can enable the teacher to provide preemptive intervention, such as delivering targeted and corrective feedback and content scaffolding to further advance their students' mathematical proficiency.

Research has shown strong connections between students' writing abilities and their capacity to learn in general (US National Institute for Literacy, 2007), to learn new mathematics content (Hayes, 1996; Meel, 1999), to improve mathematics problem-solving skills (Bicer et al., 2013; Pugalee, 2004), and to augment processes related to cognition (Flower, Schriver, Carey, Haas, \& Hayes, 1992). Gadanidis, Hughes, and Cordy (2011) found that students in middle school, who are gifted, successfully used multiple modes of communication, including drawing, writing, and an online discussion forum to learn mathematics.

Much of the research concerned with writing and mathematics is focused on journaling (Bagley \& Gallenberger, 1992; Meel, 1999) and more recently blogging (Urquhart, 2009; Zemelman, Daniels, \& Hyde, 2012). Bagley and Gallenberger (1992) proposed that journal writing serves multiple and diverse purposes; from improving students' ability to clarify and organize their thoughts to reflecting on the substance of what they are learning to providing an avenue to express their disposition and 
attitudes toward writing. Hsu (2013) found that for students in Grade 6, providing an online blogging tool enhanced their ability to communicate with one another beyond the confines of a classroom. Furthermore, he found that group discussion enabled students to tackle higher cognitivedemanding tasks, largely because this caused them to more thoroughly explain their mathematical solutions. Huscroft-D'Angelo, Higgins, and Crawford (2015) provided elementary school students, with and without learning disabilities, with a personal digital notepad and a social media blogging tool, and discovered that the students with learning disabilities were significantly more likely to use the blogging tool, whereas students without learning disabilities were more likely to use the digital notepad. Furthermore, all students improved in their ability to provide mathematical correct responses and increased their depth of mathematical explanations during the course of this intervention.

Despite this research base, currently mathematics teaching practices largely ignore writing as a tool for learning the subject; for example, 68 percent of teachers said that they did not use writing (of mathematics problems, proofs, explanations, or concepts) in the 2011-2012 school year (Purcell, Buchanan, Freidrich, 2013). This suggests that mathematics teachers may neither understand the value of the writing process for teaching and learning or they do not know how to take advantage of the multiple modes of writing.

\section{Writing and Communicating Mathematical Ideas Using Technology}

Scholars have argued that writing is technology (Haas, 2013; Havelock, 1986). Without the "tools of writing" - be they inkwells, pencil and paper, chalk and blackboard, laptops, tablets, etc. - writing is simply not possible (Haas, 2013). These writing technologies, in and of themselves, do not necessarily have the power to determine the quality or content of students' mathematical thinking or the clarity of the communication of their mathematical reasoning. They do, however, have the ability to change the way 'text' is represented (diagrams, graphs, etc.) and the quality of the visual and spatial relationship between writers (students) and the 'text' (Haas, 2013; Vygotsky, 1981). For example, multimodal technology tools, such as dictation and voice-activation tools that convert speech to print, programs that convert equations to graphs and descriptive text, and writing pens and tablets that allow students to draw pictures and write freely, have made writing easier and more accessible for all students, particularly for students with learning disabilities.

Likewise Skype, Google Hangout, Slack, and other digital technologies have made communication at a distance much simpler and more collaborative. Combining these technologies with social digital technologies, such as Google documents, blogs, polling tools, amongst others has also changed the temporal relationship (as well as further affecting the visual and spatial relationships) between writers and their audience. Students can now easily communicate with people beyond just their classmates and their teachers. Although Scardamalia and Bereiter (1994) lauded this type of open and expansive discussion because it requires writers to make their ideas and questions understandable, they also pointed out the problems with unhelpful or even erroneous information that could arise in environments that are not curated. Consequently, these issues could lead to the concomitant serious danger of students learning incorrect mathematics or developing faulty mathematical reasoning. 
Since the mid-90's, social writing and communication tools have become commonplace inside and outside the classroom. As these digital tools continually improve, so do opportunities to study mathematics using these tools in both the classroom and at a distance. This communication is largely done through video and multimodal forms of writing. Writing is used in distance learning as a means for students to ask questions, post answers, and exchange ideas with their professors and fellow students during online classes, Massive Open Online Courses (MOOCs), and other virtual options. Similarly, an industry of "digital tutors" (e.g. Chegg, 2016; Sylvan Learning, 2016; Tutor.com, 2016), in which mathematics instruction is available on demand and accessible with a mobile device or computer, has emerged. Students typically share a screen with their tutors and often use tablets or other tools to draw their ideas or write their formulas and exchange information in real time.

Nonetheless, writing has always connected people through space, time, and culture (Haas, 2013). Furthermore, writing has allowed people to build on one another's foundations to reach new insights. As Powell and Lopez (1989) maintained, writing is a heuristic tool that leads to the generation of new knowledge. What is different now is that these newer technologies enable active communication-at a distance-to take place in real time, meaning that communication in mathematics can now occur both in a synchronous and asynchronous fashion. A well-engineered discussion in a classroom can match or surpass a digital record but digital writing is not constricted by the same physical limitation as a classroom discussion. For example, writing can occur remotely from the comfort of one's bedroom. Students can collaboratively update the same document and/or discuss their ideas related to the material. These technologies, in turn, change the way the written word is recorded, making it more frequent, organic, and dynamic. In this environment, students become part of an interconnected community; as students make meaning in a digital world, their written record of thoughts has the potential to keep pace with their growing understanding in a way that pencil and paper notes cannot.

\section{Summary of the Literature Review}

The following is a summary of the relevant literature on writing in mathematics, with a focus on mathematical communication using digital technologies:

1. Writing is an active process of sense-making, in which students are required to think, question, organize, and explain their reasoning. Consensus is growing around the importance of writing as an integral part of learning mathematics and developing mathematical ways of thinking, in part due to the impetus of the US Common Core State Standards.

2. As digital technologies continually improve and enable easier communication and foster realtime collaboration, studying and learning at a distance is becoming more commonplace. Intensifying in tandem is the fundamental requirement that students learn how to communicate their mathematical knowledge and reasoning with clarity and coherence.

3. Writing is multimodal, including text, symbols, graphs, and drawings. Expressing one's self precisely through multimodal writing is an essential part of formal writing in mathematics (e.g., writing equations). Yet these various modes of expression also provide students with a more 
informal way to gain insight into abstract mathematical concepts and relationships (e.g., drawing diagrams).

4. An established evidence-base shows a strong relationship between writing and learning outcomes, including positive effects of journaling and blogging on the teaching and learning of mathematics. Nonetheless, most teachers do not use writing as a tool to teach mathematics.

5. Although different technologies may facilitate different modes of writing and processing, they do not automatically lead to cognitive understanding or clear communication. In fact, writing in unsupervised environments can lead to incorrect information, off-task interactions, and the risk of students learning wrong mathematics.

\section{Methodology}

\section{Overall Design: Two Studies of Students' Mathematical Writing}

As part of a larger research program on reading and writing mathematically sponsored by the Mathematics eText Research Center at the University of Oregon (MeTRC, 2012), two related studies examining elementary students' use of digital writing technologies were conducted. A joint research team from two universities in two states, Texas and Oregon, collaborated on the project.

Study A took place in Texas and Study B was carried out in Oregon schools. In both studies, elementary school students (ages 8-13) were required to communicate their mathematical ideas using two different technologies: a "personal digital notepad" and a "social math blog". The researchers selected these writing tools because of their prominence in the literature, particularly with regard to the study population.

The two studies shared the same research questions, employed the same online mathematics programs as the digital writing environment for the intervention, and followed similar intervention protocols, as described in the subsequent sections.

\section{Research Questions}

Four primary research questions were addressed in this study:

1. How are students using a personal digital notepad and a social math blog to communicate their mathematical ideas?

2. What types of mathematical writing are students engaging in when asked to communicate their mathematical thinking using digital technologies?

3. What types of social writing are students engaging in when asked to communicate their mathematical thinking using digital technologies? 
4. What are the similarities and differences between students' styles of communication in Study A and Study B?

\section{Digital Mathematics Intervention}

As the context for the intervention, both studies used the same online digital mathematics program, the Math Learning Companion (MLC, 2016). This program was initially designed for students in Grades 3-8, who are low performing in mathematics, as a supplemental online curriculum. MLC consists of seven modules and 73 lessons, which comprehensively cover the topics of Numbers and Operations, Algebra, Geometry, and Data Analysis. Each lesson includes six sections, which students are required to work through in order to complete the lesson:

1. Real World: an animated video that provides a real world example application of the lesson's content, which ends with an Essential Question that presents the learning objective of the lesson.

2. Vocabulary: New mathematical terms found in the current lesson are presented via an animated video and practiced with sets of questions and interactive mathematics problems.

3. Instruction: Explicitly delivered content, also presented via animated videos and practice with interactive mathematics problems.

4. Try It: Guided practice through a series of interactive mathematics problems.

5. Game: Practice of key concepts by working through a short scenario puzzle or game.

6. Final Quiz: 10 multiple choice items randomly selected from a bank of approximately 30 questions for each lesson.

Each of the six sections is divided into multiple discrete pages. Students navigate among pages by NEXT and PREVIOUS buttons. The animations can be controlled by PAUSE, PLAY, and REWIND commands. From any page, students can access digital resources including a Dictionary, a Calculator, two different Help Pages, a page of Formulas, and a Spanish Language Lesson Summary. Spoken audio, available in English and Spanish, is used in the instructional animations, to read words and definitions in the Dictionary and Formulas pages and to read questions and answers on the quiz.

The MLC curriculum framework is modeled after HELP Math (Digital Directions International, 2005), which has been shown to have a positive impact on English language learners (Freeman, 2012; Tran, 2005), as well as meeting the What Works Clearinghouse criteria in 2012.

\section{Digital Writing Environment}

In addition to the digital resources described above, MLC contains a personal digital notepad and a social math blog, which are the focus of this study. The "NotePad" (personal digital notepad) allows students to take notes directly in the program. Students can access their notes during subsequent pages and sections of the program, and can edit, delete, and flag their notes for the attention of their teacher. The social math blog, called the "Wall" allows students to post original ideas and questions for all other students to view, and to post comments on other student's posts. The intention of the blog is to promote mathematical communication through a digital group discussion. 
Both of these writing features support multiple modes of communication, allowing students to create notes/posts that involve typing, writing (via a mouse), drawing, and/or inserting mathematical symbols.

\section{Study A Intervention Protocol}

The study intervention asked each student to complete eight lessons from MLC. Students were expected to work through the six sections of each lesson, enter notes of particular types into the NotePad, or the Wall, and then to take the lesson quiz. If students did not answer at least 7 of the 10 quiz questions correctly, they repeated the lesson, although without the Real World or Games sections. The students in the grade level were assigned the same eight MLC lessons, although sometimes in different orders, and lessons chosen for each grade level were developmentally appropriate as judged by the cooperating teacher. Students were allowed to use MLC resources, such as the Dictionary, as they felt the need, except for the NotePad and the Wall, where they were required to post notes of particular types. For the first two lessons that were implemented during the study, students were asked to take at least three notes in the NotePad; specifically, students were to (1) answer the Essential Question at the end of the Real World section; (2) write one new thing they learned during the instruction and/or guided practice sections of the program; and (3) revisit up to three questions that they got wrong on the Final Quiz and explain why they missed the problem (if a student had a perfect score, then this requirement was dropped). During lessons 3 and 4 , students were tasked with submitting three posts to the social math blog. These tasks were similar to the ones for the first two lessons (answer the Essential Question and write one new mathematics idea they learned); however, the third task was to post a comment on a peer's blog post. For lessons 5 and 6, students were asked to write in their NotePad using a specific note-taking strategy intended to elicit students' reasoning within problem solving. This strategy involved having students focus on what they are learning, only write important points, use their own words, and refer to their notes later. For lessons 7 and 8, students were free to use the NotePad or the Wall in any way they chose. The purpose of this last exercise was to see if students' communication remained mathematics focused or turned to other types of social communication. All students were provided notecards to remind them of the various note taking tasks.

\section{Study B Intervention Protocol}

The general arrangements for this study were similar to Study A in that students worked through a sequence of MLC lessons, using resources as they found useful, attempted the quiz, and repeated lessons where they did not pass the quiz. There were, however, two distinct differences that were driven by differences in the educational settings of the two studies. In Study B, the students were all $6^{\text {th }}$ graders, attending the same supplemental mathematics class. Their mathematics skills varied, as did their reasons for being assigned to this class. The differences were, first, students were assigned lessons individually according to their needs, and in reference to the curriculum in the regular mathematics class each attended. Second, because the students were working at different speeds, on different lessons, the expectations for posting notes to the NotePad and to the Wall were determined by calendar periods, rather than by lessons as was done in Study A: 
- First Period: Respond to the Essential Questions and write a note during the Learn It section describing something important that was learned.

- Second Period: Using the Wall: ask a question during Learn It section, answer a question during Try It, write or draw something you learned about during the Your World section.

- Third Period: Respond to the Essential Question, write a note during the Learn It and, after a quiz, review 2 or 3 correct answers and describe why the correct answer is correct, using the NotePad.

\section{Participants}

In total, across the two studies, 42 students participated in this intervention. Participants in Study A were younger, spanning grades 3-5, while all participants in Study B were in grade 6. A complete breakdown of the demographics is shown in Table 1 . The participants are discussed separately because the school settings and the populations differed substantially between the two studies.

Table 1. Participant Demographics for Study A and Study B

\begin{tabular}{|c|c|c|}
\hline & $\begin{array}{l}\text { Study A } \\
(n=31)\end{array}$ & $\begin{array}{l}\text { Study B } \\
(n=11)\end{array}$ \\
\hline Age & $M=9.67, S D=0.85$ & $M=11.79, S D=0.30$ \\
\hline \multirow[t]{3}{*}{ Grade Level } & Grade 3: 16 students (51.6\%) & Grade 6: 9 students (100\%) \\
\hline & Grade 4: 12 students (38.7\%) & \\
\hline & Grade 5: 3 students (9.7\%) & \\
\hline \multicolumn{3}{|l|}{ Gender } \\
\hline Male & 18 (58.1\%) & $8(67 \%)$ \\
\hline Female & $13(41.9 \%)$ & $3(33 \%$ \\
\hline \multicolumn{3}{|l|}{ Race/Ethnicity } \\
\hline Caucasian & 26 (83.9\%) & $8(89 \%$ \\
\hline African American & $3(9.7 \%)$ & - \\
\hline Hispanic & -- & $1(11 \%$ \\
\hline Asian & $1(3.2 \%)$ & - \\
\hline Multiracial & $1(3.2 \%)$ & - \\
\hline Other & -- & - \\
\hline \multicolumn{3}{|l|}{ Verified Disability } \\
\hline Yes & $21(67.7 \%)$ & $7(77 \%)$ \\
\hline No & $10(32.2 \%)$ & $2(23 \%)$ \\
\hline
\end{tabular}




\section{Study A Participants}

The participants of this study attended two different types of private schools in North Texas. Thirtyone students completed the program between both schools. School 1 focused specifically on students with learning differences (as defined by the school itself), and fourteen students participated in the study, after attrition. In this school, seven of the students were in Grade 3 and eight were in Grade 4. School 2 was a private school with a general education population; nine students in Grade 3 participated, and eight students in the fourth/fifth grade class (a combined class that received lessons together) participated (fourth grade, $n=5$; fifth grade, $n=3$ ).

The sample contained several students with verified disabilities, including eleven students who were verified with learning disabilities $(n=11)$, 'Other Health Impairment' (e.g., ADHD, anxiety; $n=6$ ), autism $(n=2)$, speech/language impairment $(n=1)$, and emotional disturbance $(n=1)$.

\section{Study B Participants}

The eleven participants in Study B attended the same Oregon middle school. At this school, three types of mathematics class were available to these $6^{\text {th }}$ graders: (a) a general education mathematics class, with a curriculum that followed state and district mathematics standards, to which all students were assigned; (b) an additional supplementary mathematics class, also following the same standards, but with a greater focus on individual needs; the class was intended for students at risk of failing state mandated standardized testing, though judged likely able to pass if provided additional mathematics support; and (c) a small supplementary mathematics class for students judged unlikely to pass the state test. The Study B participants were the members of this third class.

This group contained several students with verified disabilities including specific learning disabilities $(n=4)$; emotional disturbance $(n=1)$, and other health impairment $(n=2)$.

\section{Procedures}

\section{Study Procedures}

The procedures were substantially the same in both studies. The principal investigator at each site conducted a structured training session with four researchers who then implemented the study. Training included the goals of the study, specific instructions for working with participants, an opportunity to practice administering the intervention script, and training on how to apply the intervention protocol, note-taking strategies, etc. Participants were recruited and provided consent prior to participating in the study. Teachers were asked to assess their lesson plans and assign their class a sequence of eight MLC lessons that coincided with the current curriculum. The participants completed the assigned MLC lessons while moving through the intervention tasks. Participants used the MLC program twice weekly for 45 minutes as a supplement to the regular mathematics curriculum. Because the students completed this program at their own pace, the intervention lasted ten weeks for some students and fourteen weeks for others. 
Prior to completing the lessons, participants were trained on writing in mathematics using the tools embedded within the MLC digital writing environment. Researchers introduced student participants to writing in mathematics through a scripted training specifically focused on using the features within the NotePad and the social math blog, including typing, digital writing through the use of a mouse, drawing within the program, and using specific mathematical symbols. This training also involved teaching students specific techniques for communicating their mathematical thinking, such as recording vocabulary, working through problems in small steps, and asking questions amongst others.

\section{Data Analysis}

The data analysis was the same across the two studies. Researchers had access to all participants' NotePads and the Wall postings, and were able to extract all notes and posts from the MLC database. Frequency counts were recorded each time a student clicked on the NotePad or the Wall and the type of writing modality was captured, and data were downloaded daily. The research team then analyzed each note and post separately. Participant notes from their personal digital journal and social mathematics blog posts were tabulated to provide a frequency for each type of note or post made, including the type of multimodal writing method used by participants in each study (e.g., type, draw).

All participants' personal digital notes and social mathematics blog posts were qualitatively coded and analyzed. Researchers scrutinized participants' digital notes and blog posts for substance of the content and formed several categories, and generated a coding dictionary (e.g., rubric) based on viable categories. The digital notes were then coded by where the original note was recorded (e.g. recorded on the NotePad, a comment on the Wall) and a coding dictionary was created for researchers to code students' writing entries along five additional dimensions. Figure 1 provides a detailed description of each coded category from the coding dictionary. Researchers coded $10 \%$ of randomly selected notes/posts and reached a $97.6 \%$ agreement as to the coding across categories, prior to coding independently. All disagreements in the categories were discussed amongst the researchers and a consensus was agreed prior to independent coding. 


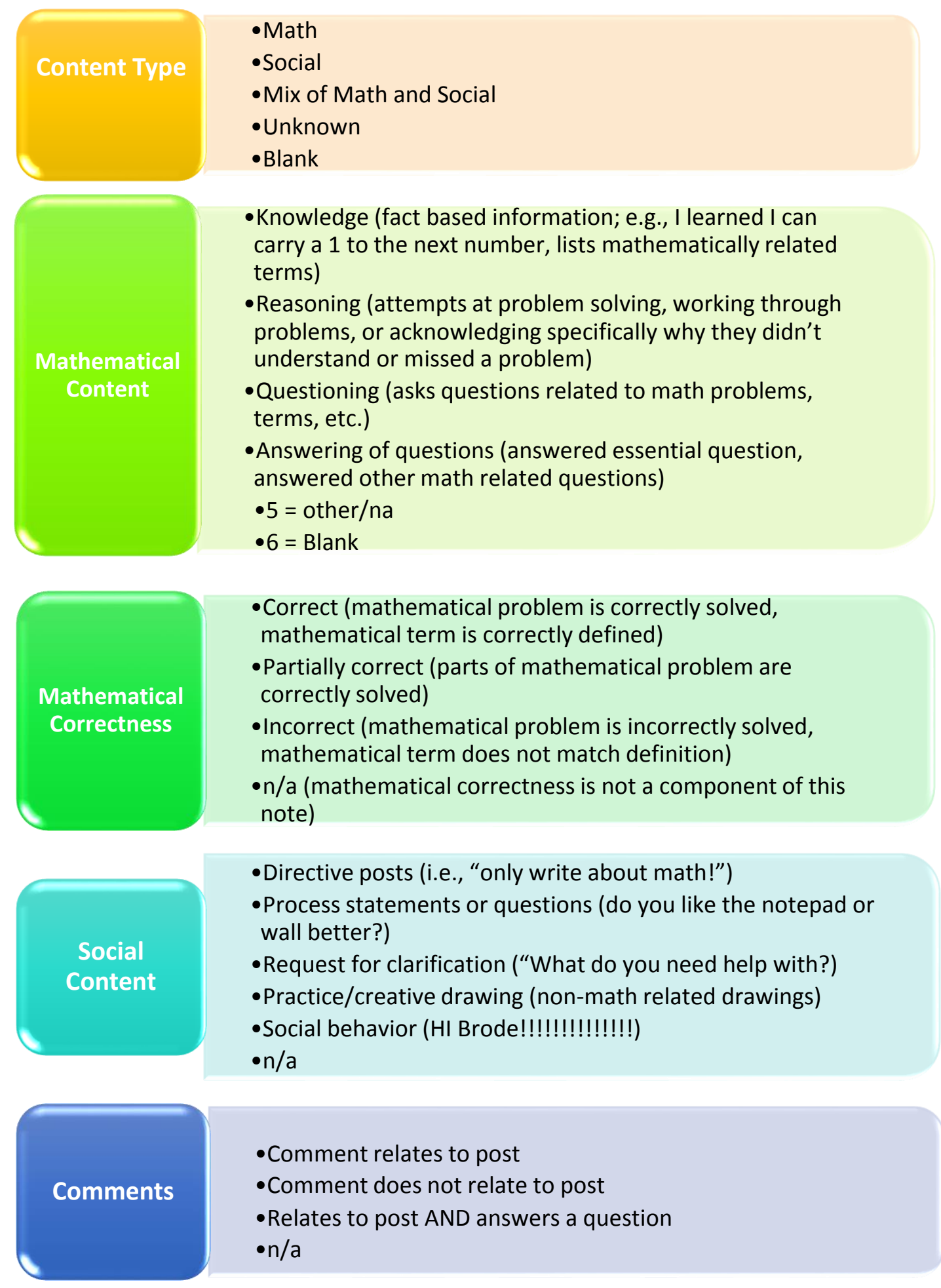

Figure 1. Coding Dictionary Definitions for Overall Content Type, Types of Mathematical Content, Assessment of Mathematical Correctness, Types of Social Content, and Assessment of the Comments. 


\section{Results}

Findings from these studies are presented as frequencies and percentages of each category with anecdotal support as organized by research question. Due to the differences in study population and procedures, the results of Study A and Study B are presented separately for research questions 1-3. Since research question 4 is a comparative question, the results of the two studies are presented together.

\section{Results - Study A}

Research Question 1: How are students using a personal digital notepad and a social math blog to communicate their mathematical ideas?

Students in Study A created a total of 705 unique entries on the NotePad, averaging 12.38 (SD = 9.62) notes per student. For the Wall, students wrote 322 individual entries $(M=10.73, S D=12.10)$, and 252 of these were original posts whereas 70 were comments on a post. On the personal digital notepad, $90.9 \%$ of notes were mathematically related; however, only $60.9 \%$ of the Wall posts were related to mathematics (see Table 2 for details).

Table 2. Frequencies and Percentages of Students' Digital Notepad and Social Blog Use as Related to Mathematical Content for Study A

\begin{tabular}{|c|c|c|}
\hline Variable & $\begin{array}{l}\text { Notepad } \\
(n=705)\end{array}$ & $\begin{array}{l}\text { Blog } \\
(n=332)\end{array}$ \\
\hline \multicolumn{3}{|l|}{ Type of Information ${ }^{a}$} \\
\hline Mathematical knowledge & $296(46.2 \%)$ & $72(36.7 \%)$ \\
\hline Mathematical reasoning & $190(29.7 \%)$ & $37(18.9 \%)$ \\
\hline Mathematical questioning & $28(4.4 \%)$ & $63(32.1 \%)$ \\
\hline Mathematical answering & $127(19.8 \%)$ & $24(12.2 \%)$ \\
\hline \multicolumn{3}{|c|}{$\begin{array}{l}\text { Notes Considered N/A for Type of } \\
\text { Information }\end{array}$} \\
\hline Other/na & $53(7.5 \%)$ & $125(37.7 \%)$ \\
\hline Blank & $11(1.6 \%)$ & $11(3.3 \%)$ \\
\hline \multicolumn{3}{|c|}{ Mathematically Correct Information ${ }^{\text {b }}$} \\
\hline Correct & $352(93.1 \%)$ & $98(88.3 \%)$ \\
\hline Partially correct & $21(5.6 \%)$ & $8(7.2 \%)$ \\
\hline Incorrect & $5(1.3 \%)$ & $5(4.5 \%)$ \\
\hline \multicolumn{3}{|c|}{ Not applicable for Mathematically Correct } \\
\hline Information & $327(46.4 \%)$ & $221(66.6 \%)$ \\
\hline
\end{tabular}


On the Wall, $16.1 \%$ of posts were exclusively social and an additional $5.3 \%$ were socially and mathematically mixed (see Table 3).

Table 3. Frequencies and Percentages for Types of Information Posted on Students' Social Blog for Study $A(N=322)$

\begin{tabular}{ll}
\hline Variable & $\mathbf{f ( \% )}$ \\
\hline Content Type & \\
Social & $52(16.1 \%)$ \\
Math & $196(60.9 \%)$ \\
Mix - social \& math & $17(5.3 \%)$ \\
Unknown & $54(16.8 \%)$ \\
Blank & $11(3.4 \%)$ \\
Type of Social Information ${ }^{\text {a }}$ & \\
Directive & $1(.9 \%)$ \\
Process statement or questions & $29(26.1 \%)$ \\
Request for clarification & $7(6.3 \%)$ \\
Practice/creative drawing & $43(38.7 \%)$ \\
Social behavior & $31(27.9 \%)$ \\
Comments & \\
Relates to post & $51(63.8 \%)$ \\
Does not relate to post & $17(21.3 \%)$ \\
Answers a question & $12(15.0 \%)$ \\
\hline
\end{tabular}

apercentages of types of social information are based on applicable notes only $(n=111)$. ${ }^{\text {bPercentages of }}$ comments based only on number of comments $(n=80)$.

\section{Research Question 2: What types of mathematical and social writing are students engaging in when asked to communicate their mathematical thinking using digital technologies?}

Figure 2 provides actual examples of the specific types of mathematical communication in which students are engaging. With reference to the mathematical coding definition provided in Figure 1 , students used the NotePad to communicate ideas around mathematical knowledge in $46.2 \%$ of their notes; however, this drops to $36.7 \%$ for the social math blog. Students were also more likely to communicate mathematical reasoning on the personal digital notepad $(29.7 \%)$ than they were on the social mathematics blog (18.9\%). Even though students were informed that their teachers and researchers had access to their writing, they asked substantially more mathematical questions on the Wall (32.1\%) than on the personal NotePad (4.4\%); however, answers to mathematical questions were more likely to be provided on the NotePad (19.8\%) than on the Wall (2.2\%). 


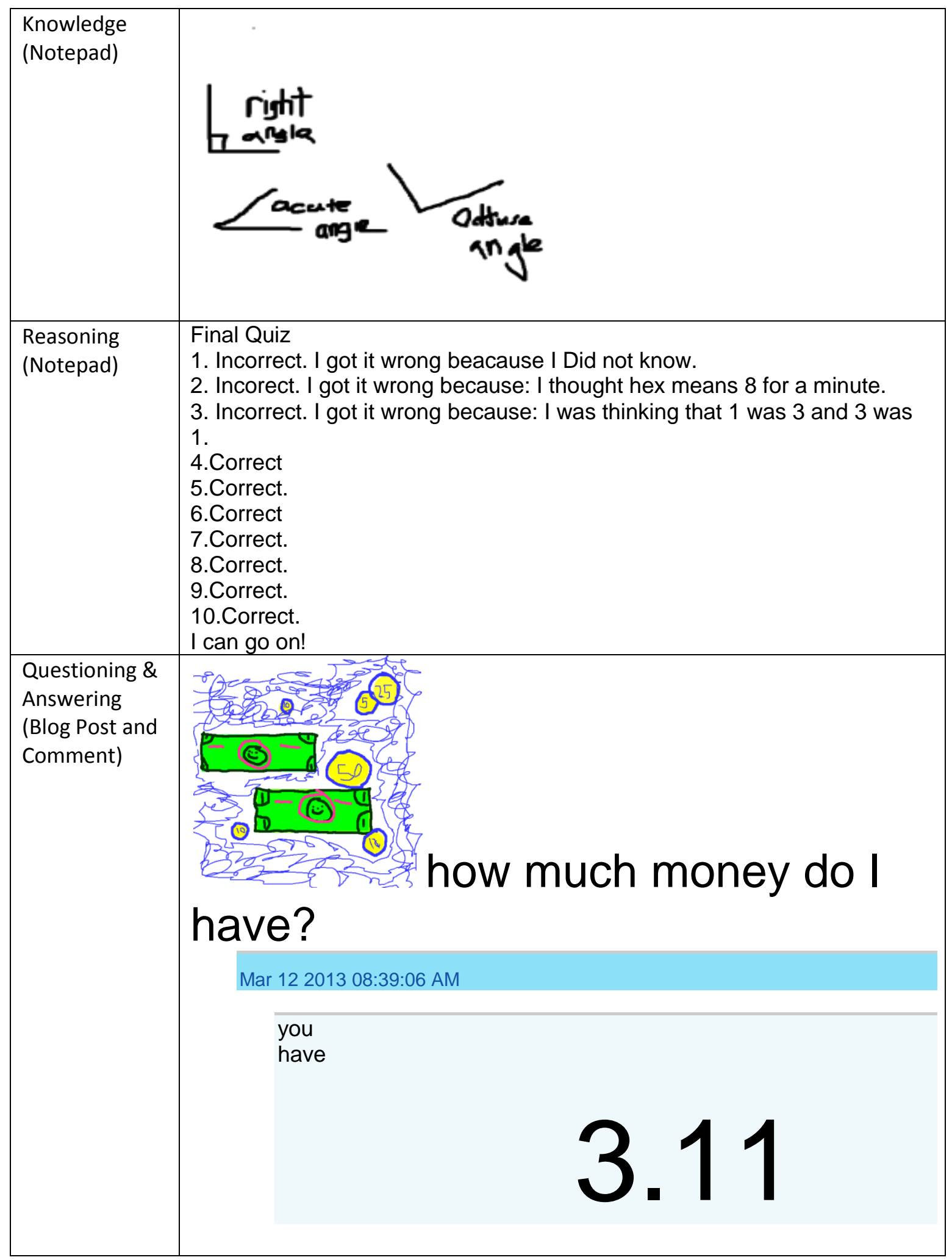

Figure 2. Student Examples of Different Types of Mathematical Communication in Study A. 
In accordance with the mathematical correctness standards provided in the coding dictionary in Figure 1, students overwhelmingly provided correct mathematical information or answers on both the NotePad (93.1\%) and the social math blog (88.3\%). Interestingly, more than half of the notes taken in the NotePad contained information that could be assessed for correctness; however, only one-third of the posts to the Wall contained information that could be assessed for mathematical correctness due to the notes/posts either containing social information or mathematical information that could not be assessed for correctness (see Figure 1 for more detail).

\section{Research Question 3: What types of social writing are students engaging in when asked to communicate their mathematical thinking using digital technologies?}

With reference to the social content definitions in the coding dictionary provided in Figure 1, students' social communications were categorized into directive, process statements or questions, requests for clarification, practice or creative drawing, or social behavior. Figure 3 provides examples of participants' social communication. Notably, over one-third of the social posts were practice or creative drawings, which may or may not have been directed at other students. Just over one-fourth of the posts were either social behavior or process statements/questions, and very few of the posts directed other students or asked questions for clarification. Students clearly used the Wall to communicate with one another; however, they also used this arena for creative expression outside of direct communication. When students commented on other students' posts, nearly twothirds of the comments related to the post itself, and an additional $15 \%$ not only gave a relevant response but actually answered a specific question asked in the post. This implies that students were engaged with the Wall and posted relevant responses to other students' questions or ideas.

\begin{tabular}{|c|c|}
\hline $\begin{array}{l}\text { Directive (Blog } \\
\text { Post and } \\
\text { Comment) }\end{array}$ & $\begin{array}{l}\text { Mar } 142013 \text { 08:22:37 Math Foundations 1, L6 Decimals \& Money, Instruction, Read } \\
\text { and Write Decimals Using Place Value } \\
\text { Hide Comment Save To My Notepad } \\
\text { Nar } 14201308: 52: 53 \text { AM } \\
\text { thate to who ever did this, } \\
\text { You only need to click it once... to save or else it will put a lot of } \\
\quad \text { P.S.Its very orginised and nice. }\end{array}$ \\
\hline $\begin{array}{l}\text { Process } \\
\text { statement or } \\
\text { questions }\end{array}$ & $\begin{array}{l}\text { Apr } 232013 \text { 07:42:11 Math Foundations 2, L4 Addition \& Subtraction, Instruction, } \\
\text { Estimating Sums and Differences } \\
\text { do you like the notepad or the wall? } \\
\text { Hide Comment Save To My Notepad }\end{array}$ \\
\hline
\end{tabular}




\begin{tabular}{|c|c|}
\hline & Apr 232013 07:46:11 AM \\
\hline & I love it! \\
\hline \multirow[t]{6}{*}{$\begin{array}{l}\text { Request for } \\
\text { clarification }\end{array}$} & $\begin{array}{l}\text { Mar } 062013 \text { 11:57:36 Math Foundations 1, L3 Multiplication, Introduction, } \\
\text { Introduction }\end{array}$ \\
\hline & Save To My Notepad \\
\hline & Apr 032013 12:07:12 \\
\hline & what is that? \\
\hline & Mar 122013 12:19:50 PM \\
\hline & Black hole \\
\hline \multicolumn{2}{|l|}{$\begin{array}{l}\text { Practice/creative } \\
\text { drawing }\end{array}$} \\
\hline Social behavior & $\begin{array}{l}\text { MULTIPACATION!!!!!!!!!!!!!!!!!!!!!!!!!!!!!!!!!!!!!!!!!!!!!!!! } \\
\text { PS. HAPPY PI DAY } \\
\text { PPS.IT DOES NOT MEAN EAT PIE IT MEANS THE } \\
\text { NUMBER PI }\end{array}$ \\
\hline
\end{tabular}

Figure 3. Student Examples of Different Types of Social Communication in Study A.

\section{Results - Study B}

Research Question 1: How are students using a personal digital notepad and a social math blog to communicate their mathematical ideas?

In Study B, students posted to the Wall more than three times as much as they wrote in their personal NotePads. Students wrote 10.21 notes on average $(S D=7.45)$ for a total of 180 notes, and they posted on average 32.37 times $(S D=24.71)$ to their social math blog for a total of 451 posts (only 23 of which were comments on a post; 428 were original posts). Students posted mathematically related information $67.8 \%$ of the time on their NotePad (see Table 4). 
Table 4. Frequencies and Percentages of Students' Digital Notepad and Social Blog Use as Related to Mathematical Content for Study B

\begin{tabular}{|c|c|c|}
\hline Variable & $\begin{array}{l}\text { Notepad } \\
(n=180)\end{array}$ & $\begin{array}{l}\text { Blog } \\
(n=451)\end{array}$ \\
\hline \multicolumn{3}{|l|}{ Type of Information } \\
\hline Mathematical knowledge & $67(54.9 \%)$ & $8(12.9 \%)$ \\
\hline Mathematical reasoning & $22(18.0 \%)$ & $3(4.8 \%)$ \\
\hline Mathematical questioning & $14(11.5 \%)$ & $30(48.4 \%)$ \\
\hline Mathematical answering & $19(15.6 \%)$ & $21(33.9 \%)$ \\
\hline \multicolumn{3}{|l|}{ Notes Considered N/A for Type of Information } \\
\hline Other/na & $53(29.4 \%)$ & $361(80.0 \%)$ \\
\hline Blank & $5(2.8 \%)$ & $28(6.2 \%)$ \\
\hline \multicolumn{3}{|l|}{ Mathematically Correct Information } \\
\hline Correct & 74(93.7\%) & $19(82.6 \%)$ \\
\hline Partially correct & $5(6.3 \%)$ & $3(13.0 \%)$ \\
\hline Incorrect & -- & $1(4.3 \%)$ \\
\hline Not applicable for Mathematically Correct Information & $101(56.1 \%)$ & $428(94.9 \%)$ \\
\hline \multicolumn{3}{|c|}{$\begin{array}{l}\text { Percentages of mathematical knowledge, reasoning, questioning, and answering are calculated based on } \\
\text { pplicable notes; therefore, for the notepad, } n=122 \text {, and for the social blog, } n=62 \text {. } \\
\text { Percentages of correct, partially correct, and incorrect are calculated based on applicable notes; therefore, } \\
\text { or the notepad, } n=79 \text {, and for the social blog, } n=23 \text {. }\end{array}$} \\
\hline \multicolumn{3}{|c|}{$\begin{array}{l}\text { On the Wall, however, only } 13.3 \% \text { of the notes contained exclusively mathematical content, an } \\
\text { another } 2.9 \% \text { of the notes were a mixture of mathematics and social content (see Table } 5 \\
\text { Therefore, over three-fourths of the posts on the Wall were socially related in Study B. } \\
\text { Table 5. Frequencies and Percentages for Types of Information Posted on Students' Social Blog for } \\
\text { Study B }(\mathrm{N}=451)\end{array}$} \\
\hline Variable & & \\
\hline \multicolumn{3}{|l|}{ Content Type } \\
\hline Social & & $8(74.9 \%)$ \\
\hline Math & & $(13.3 \%)$ \\
\hline Mix - social \& math & & $(2.9 \%)$ \\
\hline Unknown & & $(2.7 \%)$ \\
\hline Blank & & $(6.2 \%)$ \\
\hline \multicolumn{3}{|l|}{ Type of Information } \\
\hline Directive & & $.9 \%)$ \\
\hline Process statement or questions & & 7\%) \\
\hline Request for clarification & & (7.1\%) \\
\hline Practice/creative drawing & & $(5.0 \%)$ \\
\hline Social behavior & & $1(85.3 \%)$ \\
\hline
\end{tabular}




\section{Comments $^{\mathrm{b}}$}

Relates to post

$140(53.2 \%)$

Does not relate to post

$110(41.8 \%)$

Answers a question

$13(4.9 \%)$

apercentages of types of social information are based on applicable notes only $(n=423)$.

bercentages of comments based only on number of comments $(n=263)$.

\section{Research Question 2: What types of mathematical writing are students engaging in when asked to communicate their mathematical thinking using digital technologies?}

Examples of students' mathematical communication from Study B are depicted in Figure 4. With reference to the mathematical content standards in the coding dictionary (Figure 1), students wrote considerably more about mathematical knowledge and reasoning on their personal digital notepad (54.9\% related to knowledge and $18.0 \%$ contained reasoning) than on the social math blog ( $12.9 \%$ contained knowledge and $4.8 \%$ used reasoning). As expected, students asked and answered substantially more questions on the Wall, where $48.4 \%$ of mathematical posts were questions and $33.9 \%$ were answers, than on the NotePad, where $11.5 \%$ of posts were questions and $15.6 \%$ were answers.

\begin{tabular}{|c|c|}
\hline \multirow{2}{*}{$\begin{array}{l}\text { Knowledge } \\
\text { (Notepad) }\end{array}$} & May 162013 11:19:52 AM Flag Share On Wall \\
\hline & $\begin{array}{l}\text { Math Foundations } 3, \text { L7 Add, Subtract, Multiply, \& Divide Decimals, Vocabulary, } \\
\text { Regroup } \\
2 \text { pints }=1 \text { quart } \\
4 \text { quarts }=1 \text { gallon } \\
\text { for example } 13473+3992=17465 \text { all i did was } 4009+11303=1393 \\
\text { if the first number is larger in quantity you divide if the first number is } \\
\text { smaller in quantity then you multiply }\end{array}$ \\
\hline \multirow{2}{*}{$\begin{array}{l}\text { Reasoning } \\
\text { (Notepad) }\end{array}$} & May 142013 11:28:23 AM Flaq Share On Wall \\
\hline & $\begin{array}{l}\text { Numbers Make Sense, L6 Working with Decimals and Percents, Final Quiz, Page } \\
1 \\
\text { to figure the total cost first get the ammount of tax by changing the } \\
\text { percent to decimal and muliply } 12.00 \text { by the decimal then add. }\end{array}$ \\
\hline \multirow{2}{*}{$\begin{array}{l}\text { Questioning \& } \\
\text { Answering (Blog } \\
\text { Post and Comment) }\end{array}$} & May 092013 11:28:18 AM Flag \\
\hline & $\begin{array}{l}\text { Numbers Make Sense, L6 Working with Decimals and Percents, Try lt, Question } \\
8 \\
\text { A bike is discounted } 15 \% \text {. the original price } \\
\text { of the bike is } \$ 175 \text {. whats the discounted } \\
\text { price? } \\
\text { can you help me with this problem please? } \\
\text { Hide Comment }\end{array}$ \\
\hline
\end{tabular}




\begin{tabular}{|l|l|}
\hline & May 09 201311:30:32 AM \\
\hline i can well you need to subtract 15\% from the original price \\
\hline
\end{tabular}

Figure 4. Student Examples of Different Types of Mathematical Communication in Study B

When notes/posts contained analyzable mathematical information, student responses were correct or partially correct $100 \%$ of the time on the personal digital notepad and $95.6 \%$ of the time on the social math blog. Only half of the notes on the NotePad were able to be assessed for correctness, and only $5.1 \%$ of the posts $(n=23)$ on the Wall were able to be assessed due to the notes/posts either containing social information or mathematical information that could not be assessed for correctness (see Figure 1 for more detail).

\section{Research Question 3: What types of social writing are students engaging in when asked to communicate their mathematical thinking using digital technologies?}

With reference to the social content definitions provided in the coding dictionary in Figure 1 , in Study $B$, students used the Wall to communicate non-mathematical information on a regular basis, with $85.3 \%$ of the posts involving simple social behavior (e.g., telling a friend "Hi," writing "epic face" accompanied by a drawing; see Figure 4 for a comment thread). Of the remaining notes, 7.1\% involved requests for clarification, $5.0 \%$ contained a creative drawing or practice, $1.9 \%$ were directives, and $0.7 \%$ were process statements or questions. Interestingly, in Study B students posted more comments to other posts than original posts (comments, $n=263$; original posts, $n=188$ ). A little over half of these comments directly related to the original post, and an additional 5.9\% answered a specific question. As shown in Figure 5, students in Study B used the Wall to primarily communicate with peers about non-mathematically related information, and much of this communication was reciprocal given the high number of comments related to initial posts.

\begin{tabular}{|l|l|}
\hline Directive & $\begin{array}{l}\text { May 09 2013 11:45:10 Numbers Make Sense, L6 Working with Decimals and } \\
\text { Percents, Try It, Question 8 }\end{array}$ \\
\hline $\begin{array}{l}\text { you are being annoying with your behavior. } \\
\text { or questions }\end{array}$ & $\begin{array}{l}\text { May 14 2013 11:44:05 Math Foundations 2, L2 Fractions \& Decimals, Instruction, } \\
\text { Naming Fractions Practice } \\
\text { anyone need help ? } \\
\text { Hide Comment Save To My Notepad }\end{array}$ \\
\hline $\begin{array}{l}\text { May 16 2013 11:07:28 AM } \\
\text { not at the moment but thank you for asking }\end{array}$ \\
\hline
\end{tabular}




\begin{tabular}{|l|l|}
\hline $\begin{array}{l}\text { Request for } \\
\text { clarification }\end{array}$ & $\begin{array}{l}\text { May 14 2013 11:16:20 Numbers Make Sense, L6 Working with Decimals and } \\
\text { Percents, Vocabulary, Place Value } \\
\text { what do u need help with } \\
\text { Hide Comment Save To My Notepad }\end{array}$ \\
\hline $\begin{array}{l}\text { Practice/creative } 16201311: 30: 48 \text { AM } \\
\text { drawing }\end{array}$ & $\begin{array}{l}\text { Apr } 30201311: 38: 04 \text { Numbers Make Sense, L6 Working with Decimals and } \\
\text { Percents, Vocabulary, Interest, Principal, Simple Interest }\end{array}$ \\
\hline Social behavior & $\begin{array}{l}\text { i just wanted to test out the font } \\
\text { Comments Save To My Notepad }\end{array}$ \\
\hline $\begin{array}{l}\text { May 16 2013 11:16:49 Numbers Make Sense, L6 Working with Decimals } \\
\text { and Percents, Instruction, Percent and Decimals Practice }\end{array}$ \\
\hline $\begin{array}{l}\text { Whats up im back } \\
\text { Hide Comment Save To My Notepad }\end{array}$ \\
$\begin{array}{l}\text { Mes so you are from wear!!!!!!!!!! } \\
\text { So13 11:26:38 AM }\end{array}$ \\
\hline
\end{tabular}

Figure 5. Student Examples of Different Types of Social Communication in Study B. All Examples Are Posts on the Blog and/or Comments on A Post.

\section{Research Question 4: What are the similarities and differences between students' styles of communication in Study A and Study B?}

Study A and Study B used the same intervention program and provided students with the same technology. Students in Study A used the personal digital notepad more than twice as often as they used the social math blog (NotePad, $n=705$; Wall, $n=332$ ). However, students in Study B used the Wall more than twice as often as they used the NotePad (NotePad, $n=180$; Wall, $n=451$ ). This overall difference in the usage of the personal digital notepad and the social math blog may be due to several factors, including the student population (older versus younger students), the school setting (public middle school versus personal elementary school), or due to the implementation of the intervention. 
Students predominately used the typing or text feature in both studies and across the NotePad and the Wall. Although the younger students in Study A used the draw (or the draw in conjunction with text or symbols) in the social math blog $32 \%$ of the time as compared to the older students who drew only $11 \%$ of the time on the blog. The types of multimodal writing techniques are presented in Table 6 for both studies.

Table 6. Multimodal Writing Methods for Study A and Study B on the Private Notepad and Social Blog

\begin{tabular}{lrrrr}
\hline & \multicolumn{2}{c}{ Study A } & \multicolumn{2}{c}{ Study B } \\
\hline \multicolumn{1}{c}{ Notepad } & \multicolumn{1}{c}{ Blog } & \multicolumn{1}{c}{ Notepad } & \multicolumn{1}{c}{ Blog } \\
\multicolumn{1}{c}{$(n=705)$} & \multicolumn{1}{c}{$(n=332)$} & \multicolumn{1}{c}{$(n=180)$} & \multicolumn{1}{c}{$(n=451)$} \\
\hline Typed & $529(75.0 \%)$ & $189(56.9 \%)$ & $122(67.8 \%)$ & $354(78.5 \%)$ \\
Typed \& use of symbols & $66(9.4 \%)$ & $24(7.2 \%)$ & $31(17.2 \%)$ & $18(4.0 \%)$ \\
Draw & $56(7.9 \%)$ & $72(21.7 \%)$ & $14(7.8 \%)$ & $31(6.9 \%)$ \\
Type \& draw & $38(5.4 \%)$ & $30(9.0 \%)$ & $7(3.9 \%)$ & $19(4.2 \%)$ \\
Type, draw, \& symbols & $5(0.7 \%)$ & $6(1.8 \%)$ & $1(.6 \%)$ & $1(.2 \%)$ \\
Blank & $11(1.6 \%)$ & $11(3.3 \%)$ & $5(28.0 \%)$ & $28(6.2 \%)$ \\
\hline
\end{tabular}

When communicating mathematical ideas, students behaved similarly across both studies. As expected in both studies, students used the personal digital notepad to communicate more mathematical information and the social math blog to communicate both mathematical and social information. Students wrote notes about mathematical knowledge and reasoning on the NotePad and asked more mathematical questions on the Wall. Interestingly, students in Study A were more likely to answer questions posed by other students on the Wall by responding privately in their own NotePad than publicly on the Wall. This contrasts with students in Study B who answered more questions on the Wall (the intervention protocol required that students respond to other students' queries, but it did not specify whether they should respond in their journal or on the Wall). When the notes/posts could be assessed for mathematical correctness, students in both studies displayed an overwhelming tendency to post correct information.

The Wall was used very differently in the two studies. In Study A, only $16.1 \%$ of the content on the blog was exclusively socially related, whereas $74.9 \%$ of the Wall was exclusively social in Study B. When broken into specific types of social information, students in Study A posted more creative drawings or practice, with process statements or questions and social behavior accounting for slightly over one-fourth of the Wall content each. In contrast, students in Study B, posted an overwhelming number (85.3\%) of social behavior-related posts.

When considering the comments on the NotePad, $24.8 \%$ of the posts on the NotePad were comments on another student's post in Study A, whereas $58.3 \%$ of the posts on the NotePad in Study B were comments. Students in Study A posted comments that were relevant or answered questions $78.5 \%$ of the time; however, students in Study B posted relevant information or answered questions in $58.1 \%$ of their comments. The students engaging in more social behavior were less likely to post information directly relevant to the original post than students engaging in either more creative drawing or who were asking questions for clarification. 


\section{Summary of Results}

The primary aim of this study was to better understand the influence of digital technologies on the communication of mathematical ideas through multimodal writing. Research Question 1 asked: How are students using a personal digital notepad and a social math blog to communicate their mathematical ideas? The study found that the younger students in Study A (grades 3-5) took substantially more notes on the NotePad, while the older students in Study B (grade 6) posted notes on the Wall more than twice as often as they wrote in the NotePad.

Research Question 2 asked: What types of mathematical writing are students engaging in when asked to communicate their mathematical thinking using digital technologies? In both studies, students used the digital NotePad substantially more than the Wall to communicate math knowledge and reasoning. Also, in both studies, when answers could be assessed for mathematical correctness, they were nearly always correct; however, in Study B, the number of posts eligible for assessment on the Wall was small.

Research Question 3 asked: What types of social writing are students engaging in when asked to communicate their mathematical thinking using digital technologies? In Study A, students' social writing was predominately comprised of practice and creative drawings or process statements. In contrast, in Study B, most communication was related to social behavior.

Research Question 4 asked: What are the similarities and difference between students' styles of communication in Study A an Study B. Findings indicate that students are using the NotePad and the Wall differently from one another, and illustrate that different kinds of writing technologies do have the power to influence the types of writing that students are engaging in when asked to communicate their mathematical ideas (Haas, 2013). When writing in the NotePad, students communicated more mathematical knowledge and reasoning and fewer mathematics questions and answers. Particularly in Study A, students were more likely to personally ask and answer their own mathematics questions in their NotePad than ask or respond to questions in a group discussion taking place on the Wall. When using their NotePad, students in both studies tended not to engage in much social interaction-neither on task (asking the teacher questions) nor off task (writing unrelated to mathematics) - even though they knew that the NotePad could be accessed by their teacher. When using the Wall, however, students across studies engaged in both social and mathematical communication. Students asked and answered mathematical questions collaboratively with their peers and explained their solutions to one another. This discovery reinforces Hsu's (2013) finding related to the positive effect of online blogging. Nonetheless, when writing on the Wall, students communicated less mathematical knowledge and reasoning and engaged in more non-mathematical social communication.

Furthermore, students across both studies behaved similarly to one another when writing in the personal digital notepad and much differently when communicating using the social math blog. The intervention protocol required students to complete certain mathematical tasks at each level of the intervention, which could explain some of the similarities in the types of communication of 
mathematical ideas (e.g., explaining the answer to the MLC lesson's Essential Question, reflecting on why they got answers wrong in the Final Quiz). Students, however, were not instructed to use either the personal digital notepad or the social math blog to communicate in any way other than mathematically; thus, the social behavioral communication manifested spontaneously from the students themselves (which wasn't a great surprise). In Study B, students more often engaged in communication related to social behavior (e.g., messing around, chatting; see Figure 5). Yet, students in Study A more often used their imagination and produced creative drawings and practices over other types of social interaction (see Figure 3).

\section{Discussion}

The primary aim of this study was to better understand the influence of digital technologies on the communication of mathematical ideas through multimodal writing. The studies were conceived in order to explore the interrelationship amongst writing, mathematics, and digital technologies, and to assess the influence of the intertwined components on students' communication of their mathematical ideas.

The results of the overall study suggests that the informality of writing in a social math blog or personal notepad may help some young learners get over the challenges of mathematical writing in a formal setting (e.g., on a mathematics test, homework). In both studies, when students wrote multimodal notes and posts related directly to mathematics that could be assessed for correctness, their answers were overwhelmingly accurate (see Figure 1). The quality of the writing was often poor in these posts (even at the appropriate standard for the grade level), however, the students provided mathematically correct explanations that could be understood by their peers. This implies that it was not only the highest-performing students that were providing the correct answers on the blog but rather students of all abilities were contributing (as required by the intervention) and getting their answers right. This finding lends additional support to a body of research that shows that writing in mathematics can induce students to think logically and explain their thought process (Bicer et al., 2013; Devlin, 2012; NCTM, 2014; Pugalee, 2004). Moreover, providing digital writing environments can enhance the learning process for students who may have learning disabilities (Huscroft-D'Angelo et al., 2015), as well as for students who are gifted (Gadanidis et al., 2011). Many students, particularly in Study A (the younger students), also relayed their mathematical understanding through the drawing of pictures. These pictures were not sophisticated, yet they reveal younger students' attempt to demonstrate their understanding, as well as their ability to visualize mathematics as something more than just numbers or formulas on a page.

This study indicates that multimodal writing may play an important role in helping young students acquire deeper mathematical understanding, thus helping to surmount many of the difficulties often associated with learning the subject. It can also help them overcome difficulties identified with more formal mathematical writing (Ash, n.d.). In particular, it may enable learners to overcome the barrier of the formal and exacting (Devlin, 2012) and often information-dense language of mathematics (Schleppegrell, 2007). Dewey (1997) said that "foreign subject-matter transformed through thinking into a familiar possession becomes a resource for judging and assimilating foreign subject matter" (p.223). Multimodal writing (e.g., visual representation of concepts through 
drawing) could facilitate this spiral process of content acquisition in mathematics. An analogy with learning a foreign language illuminates this idea.

The early production stage of learning a foreign language is necessarily fraught with errors; grammatical mistakes, misspelling, limited vocabulary, inaccurately recalled word meanings, and so forth (Krashen, 1987; Robertson \& Ford, 2009). This stumbling incoherent communication may look messy, yet it is a necessary staging point on the journey to mastery - and a fundamental requirement so that eventually one can communicate with people who speak another language. Likewise, early-stage writing in mathematics may look messy, but it too is a necessary scaffold toward mastery. A low-anxiety environment such as that provided by the use of digital writing tools may therefore provide an arena in which students are more willing to actively think through mathematical concepts, because they are less afraid of making mistakes when writing in their personal journal or when chatting with peers on a social blog. Furthermore, when writing in a blog, learners' mathematical writing output is interspersed with social conversation and interaction, so learners tend to be less worried about being corrected or put down for trying. This type of empathetic communication, as in language acquisition, can help students gain proficiency (Krashen, 1987). Multimodal writing in a digital environment may also serve as a forum for some students to imagine and experiment with mathematical ideas, in ways that more conventional methods of teaching sometimes do not encourage (Dill, Freeman, Frazier, \& Benito, 2015).

Nevertheless, the results of this study suggest caution when requiring students to write about mathematics without close guidance and without formally teaching them the types and range of multimodal writing that can be performed using digital tools. This caution arises from the observations in this study that the majority of students' communication was still off task, despite the fact that this study took place in a controlled environment. As Scardamalia and Bereiter (1994) observed with foresight, erroneous or irrelevant information could occur in a setting that is not monitored and guided by an expert. Thus, guided writing and discussion is essential to produce desired learning outcomes and remains a cornerstone of good teaching and learning; many developed frameworks exist to support students as they become independent writers and learners (Gibson, 2008; Gould, 2008).

As discussed in the literature review, the majority of teachers do not use writing as a tool to teach mathematics (Purcell et al., 2013). Every discipline from literature and composition to social studies and physics dictates a particular set of writing conventions. In the absence of being taught such conventions, students internalize a set of rules about what is appropriate and inappropriate for learning an academic subject. For example, although students in this study were presented with multiple modes of communication, most students chose to type their responses on both the personal digital notepad and the social math blog for the majority of the notes/posts they produced. Interestingly, however, the younger students in Study A used the drawing feature on the social math blog over three times more often than the older students in Study B. As this study indicates, with each successive grade students may become more resistant to the idea that writing about mathematics is a legitimate and important means to acquire understanding (US NGA \& US CCSSO, 2010). The earlier that writing about mathematics is introduced to students, the more normal will it seem. The earlier students are encouraged to draw and experiment with mathematics through multimodal writing, the greater the chance that they will see the patterns in mathematics and not just formulas and rules to be memorized. A high school student asked to write about mathematics 
having never had to do so before may find it practically a difficult exercise and question the purpose. On the contrary, a high school student who has written about mathematics since elementary school is more likely to view it as an appropriate practice and may even find it difficult to learn mathematics without the scaffold of writing. We saw glimmers of this effect in our study when comparing the communication patterns of the younger students in Study A (grades 3-5) with the older students in Study B (grade 6). Students need to be taught from a young age how to take advantage of digital tools to write effectively and imaginatively in mathematics, and teachers need to be better trained in how to teach students how to do so (Purcell et al., 2013).

\section{Summary of Study Results and Recommendations}

The following is a summary of the results of the study with reference to the major takeaways from the literature review. The researchers make a recommendation associated with each of these key findings.

1. The study showed that when students wrote mathematical notes that could be assessed for correctness, their answers were predominantly accurate. This confirms that actual mathematical reasoning and sense-making, or at least communication of one's already acquired mathematical understanding, is indeed taking place through writing using digital tools, as articulated in the literature. Based on the study results, the researchers recommend that, at a minimum, teachers use collaborative tools (e.g., a digital student mathematics journal that teachers can access or a mathematics blog that the teachers can moderate), which enable more organic and dynamic communication with their students. These tools can be used by teachers to gain an early awareness of their students' understanding and misconceptions, and create an opportunity to provide loops of targeted feedback to students and proactive real-time intervention.

2. The study demonstrated that different digital writing technologies have the power to influence the types of writing students engage in when communicating their mathematical thinking. It showed that students were more likely to communicate math knowledge and reasoning in their personal NotePad, yet more prone to ask and answer questions when blogging. Based on this finding, the researchers recommend that teachers carefully consider the learning outcome that they are seeking and select the digital writing tool that best fits their objectives.

3. The younger students in Study A were more likely to draw pictures to represent their mathematical thinking, than the older students in Study B. Although the pictures were not complex, they showed that multimodal writing can help students visualize and gain insight into abstract concepts and relationships. Based on this finding, the researchers recommend that students are taught from an early age how to write in mathematics (as distinct from writing in language arts or other subject areas), and teachers need to be trained in how to use multimodal tools to help their students learn. It is also important that students are taught to visualize math concepts and that their mathematical ideas can be expressed in multiple ways using writing tools (e.g., pictures, graphs, symbols, etc.). 
4. This study found that students wrote mathematical ideas that could be understood by their peers and inspired other students to engage in dialogue about mathematics. This suggests that the informality of writing in a journal or on a blog may provide a staging point on the way toward mastery by enabling students to write in a non-threatening environment in which they are less worried about making mistakes and more willing to think actively about mathematical ideas. As in point 1 , the researchers recommend that teachers use interactive writing tools to gain an insight into their students' mathematical understanding and development.

5. When writing on the social math blog, off-task conversation predominated and writing was typically unconventional, which indicates that writing is unlikely to lead to systematic improvement when students are left to their own devices. The researchers recommend that the learning environment is carefully monitored and moderated, and that students' writing, regardless of the technology, are closely guided by teachers with expertise in mathematics.

\section{Limitations}

These studies contain several limitations. Both of the studies use a small sample of students, and all of the notes or posts were written in the context of a specific mathematics intervention. Moreover, students attempted to follow a specific intervention protocol; thus, some of the notes related to communicating mathematical ideas were required by the program. Future research could examine students' mathematical and social communication on a larger population of students of varying ages and without the constraints of a specific online intervention. Although the authors draw comparisons between the studies, the similarities and differences between the types of communication presented in Study A and Study B need to be cautiously interpreted as well due to the differences in the student populations and the intervention protocol.

\section{Conclusion}

Writing in mathematics is firmly established in the US K-12 curriculum standards, and is generally understood to be a vital tool to help students both develop mathematical ways of thinking and communicate their mathematical reasoning effectively. As digital writing technologies continue to improve and enable active communication in real time, studying and learning using these tools, in the classroom and at a distance, is becoming the norm.

This research study demonstrated that using digital writing tools can both advance students' thinking and help them communicate their mathematical ideas; skills that are symbiotic yet distinct. These findings contribute to a strong theoretical basis that demonstrates the merits of multimodal writing in mathematics. In order to realize genuine impact, teachers need to be trained on how to use multimodal writing and digital writing tools to realize their learning objectives for students. Before this will happen, however, we suspect that teachers must fully appreciate the urgency of the demand for students to become independent learners in a school context and beyond, and furthermore, be convinced of the role that writing can play in helping students study and learn mathematics independently. 
There is a strong literature related to the importance and development of mathematical discourse in math classrooms; discourse meaning mathematical talk among students. The next step is to move this discourse into the written forms needed for mathematical communication when students are working at a distance, in either time or space, from their teachers and their peers.

\section{References}

Ash, R. (n.d.). Remarks on expository writing in math. Retrieved on 28 September 2016 from http://www.math.uiuc.edu/ r-ash/Remarks.pdf.

Ashlock, B. (2006). Error patterns in computation: Using error patterns to improve instruction. Upper Saddle River, NJ: Prentice Hall.

Bagley, T. \& Gallenberger, C. (1992). Assessing students' dispositions: Using journal to improve students' performance. Mathematics Teacher, 85, 660-663.

Ball, D. (1994, November). Developing mathematics reform: What don't we know about teacher learning-but would make good working hypothesis? Paper presented at the Conference on Teacher Enhancement K-6, Arlington, VA.

Baxter, J., Woodward, J., and Olson, D. (2005). Writing in mathematics: An alternative form of communication for academically low-achieving students. Learning Disabilities Research \& Practice, 20(2), 119-135.

Bicer, A., Capraro, R., \& Capraro, M. (2013). Integrating writing into mathematics classrooms to increase students' problem solving skills. International Online Journal of Educational Sciences, 5(2), 361-369.

Bruner, J. S. (1968). Toward a theory of instruction. New York: W.W. Norton.

Burns, M. (2004). Writing in math. Educational Leadership, 62(2), 30-33.

Burns, M. (1995). Writing in math class: A resource for grades 2-8. Sausalito, CA: Math Solutions.

Chegg, Incorporated. (2016). Chegg. Retrieved on 1 October 2016 from http://www.chegg.com/

Cummins, J. (1979). Cognitive/academic language proficiency, linguistic interdependence, the optimum age question and some other matters. Working Papers on Bilingualism, 19, 121 129. Retrieved on 1 October 2016 from http://www.iteachilearn.com/cummins/ bicscalp.html

Devlin, K. (2012). Introduction to mathematical thinking. Palo Alto, CA: Keith Devlin.

Dewey, J. (1997). Experience and education. New York: Simon and Schuster.

Digital Directions International (2005). HELP Math. Retrieved on 1 October 2016 from http://www.helpprogram.net/home.htm

Dill, K., Freeman, B., Frazier, S., \& Benito, J. (2015, Dec.). Mars Game: Creating and evaluating an engaging educational game. Interservice/Industry Training, Simulation, and Education Conference (I/ITSEC) 2015. Location 2015 Paper No. 15105. 
Emig, J. (1977). Writing as a mode of learning. College Composition and Communication, 28, 12228.

Flower, L., Schriver, K., Carey, L., Haas, C. \& Hayes, J. (1992). Planning in writing: The cognition of a constructive process. Technical Report 34, Center for the Study of Writing, Carnegie Mellon University, Berkeley, CA \&, Pittsburg, BA.

Freeman, B. (2013, February 15). Fluent in math. Ed Tech Digest. Retrieved on 1 October 2016 from http://edtechdigest.wordpress.com/2013/02/15/fluent-in-math/

Freeman, B. (2012). Using digital technologies to redress inequities for English Language Learners in the English speaking mathematics classroom. Computers \& Education, 59(1), 50-62. doi: 10.1016/j.compedu.2011.11.003

Gadanidis, G., Hughes, J., \& Cordy, M. (2011). Mathematics for gifted students in an arts-and technology-rich setting. Journal for the Education of the Gifted, 34(3), 397-433.

Gibson, S.A. (2008, December). An effective framework for primary-grade guided writing instruction. The Reading Teacher, 62(4), 324-334.

Gould, P (2013). Teaching mathematical reasoning: The challenge of the new syllabus. Retrieved on 1 October 2016 from https://eis.uow.edu.au/content/groups/public/@web/@inf/ @math/documents/doc/uow152422.pdf

Gould, P (2008, August). Communicating mathematical reasoning: More than just talking. Paper presented at APEC-KHON KAEN International Symposium, KhonKaen, Thailand.

Haas, C. (2013). Writing technology: Studies on the materiality of literacy. New York, NY: Routledge.

Havelock, E. A. (1986). The muse learns to write. New Haven, CT: Yale University Press.

Hayes, J. R. (1996). A new framework for understanding cognition and affect in writing. In C. M. Levy \& S. Ransdell (Eds.), The science of writing: Theories, methods, individual differences, and applications (pp. 1-27). Mahwah, NJ: Erlbaum.

Hayes, J. R. (1996). A new framework for understanding cognition and affect in writing. In C.

Hsu, W. (2013). Examining the types of mathematical tasks used to explore the mathematics instruction by elementary school teachers. Creative Education, 4(6), 396-404.

Huscroft-D’Angelo, J., Higgins, K., \& Crawford, L. (2016). Technology, affective characteristics and mathematics: A review of the literature from 1985-2013. Manuscript submitted for publication.

Huscroft-D’Angelo, J., Higgins, K., \& Crawford, L. (2015). Communicating mathematical ideas in a digital writing environment: The impacts on mathematical reasoning for students with and without learning disabilities. Social Welfare, 4(2), 68-84.

Kenney, J. M., Hancewicz, E., Heuer, L., Metsisto, D., \& Tutttle, C. L. (2005). Literacy strategies for improving mathematics instruction. Alexandria, VA: ASCD.

Krashen, S. D. (1987). Principles and practice in second language acquisition. Upper Saddle River, NJ: Prentice-Hall International. 
Lee, K. (2010). A guide to writing mathematics. Retrieved on 1 October 2016 from http://web.cs. ucdavis.edu/ amenta/w10/writingman.pdf.

Li, Q. \& Ma, X. (2010). A meta-analysis of the effects of computer technology on school students' mathematics learning. Educational Psychology Review, 22, 215-243. doi 10.1007/s10648010-9125-8

Math Learning Companion (2016). Retrieved on 1 October 2016 from http://ansersinstitute.tcu. edu/project/math-learning-companion/

Mathematics eText Resaerch Center. (2012). Center for Advanced Technology in Education. University of Oregon. Retrieved on 1 October 2016 from http://metrc.uoregon.edu/

Meel, D. (1999). Email dialogue journals in a college calculus classroom: A look at the implementation and benefits. Journal of Computers in Mathematics and Science Teaching, 18(4), 387-413.

Ong, W.J. (1982). Orality and literacy: The technologizing of the word. Routledge. New York: New York.

Oregon Department of Education (2012). Overview of Claims and Evidence for CCSS Mathematics Assessment. Retrieved on 4 November 2014 from http://www.ode.state.or.us/search/page/ ?id=3618

Powell, A.B., \& Lopez, J.A. (1989). Writing as a vehicle to learn mathematics: A case study. In P. Connolly \& T. Vilardi (Eds.), Writing to learn mathematics and science (pp. 157-177). New York: Teachers College Press.

Pugalee, D. (2004). A comparison of verbal and written descriptions of students' problem-solving processes. Educational Studies in Mathematics, 55, 27-47.

Purcell, K., Buchanan, J., \& Friedrich, L. (2013, July). The impact of digital tools on student writing and how writing is taught in schools. Retrieved on 1 October 2016 from http://www.nwp. org/afnews/PIP_NWP_Writing_and_Tech.pdf

Robertson, K. \& Ford, K. (2009). Retrieved on 1 October 2016 from http://www.colorincolorado. org/article/language-acquisition-overview

Scardamalia, M., \& Bereiter, C. (1994). Computer support for knowledge-building communities. The journal of the learning sciences, 3(3), 265-283.

Schleppegrell, M. J. (2010). Language in mathematics teaching and learning: A research review. In J. Moschkovich (Ed.), Language and mathematics education: Multiple perspectives and directions for research (pp. 73-112). Charlotte, NC: Information Age Publishing, Inc.

Schleppegrell, M. J. (2007). The linguistic challenges of mathematics teaching and learning: A research review. Reading and Writing Quarterly, 23, 139-159.

Slavin, R. \& Lake, C. (2008). Effective programs in elementary mathematics: A best-evidence synthesis. Review of Educational Research, 78, 427-515.

Slavin, R. E., Lake, C., \& Groff, C. (2009). Effective programs in middle and high school mathematics: A best-evidence synthesis. Review of Educational Research, 79(2), 839-911. 
Sylvan Learning (2016). Sylvan Learning. Retrieved on 1 October 2016 from http://www. sylvanlearning.com/

Tran, Z. (2005). Help with English Language Proficiency "HELP" program evaluation of sheltered instruction multimedia lessons [White paper]. Retrieved on 1 October 2016 from http://www.helpprogram.net/home.htm

Tutor.com, Incorporated (2016). Retrieved on 1 October 2016 from http://www.tutor.com/

Urquhart, V. (2009). Using writing in mathematics to deepen student learning. Denver, CO: Midcontinent Research for Education and Learning. Retrieved on 1 October 2016 from http://www.mcrel.org/products-and-services/products/product-listing/01_99/product19\#sthash.qy8kOKiw.dpuf

US National Council of Teachers of Mathematics (2014). Principles to actions: Ensuring mathematics success for all. Reston, VA: The National Council of Teachers of Mathematics, Inc.

US National Governors Association Center for Best Practices \& Council of Chief State School Officers. (2010). Common core state standards for mathematics. Washington, DC: Authors.

US National Institute for Literacy (2007). What content-area teachers should know about adolescent literacy. Washington DC: Author.

Vygotsky, L. (1981). The instrumental method in psychology. In J. V. Wertsch (Ed.), The concept of activity in Soviet psychology (pp. 134-143). Armonk, NY: M. E. Sharpe.

Vygotsky, L. (1986). Thought and language (Revised edition). Cambridge, MA: MIT Press.

Wiliam, D. (2011). Embedded formative assessment. Bloomington, IN: Solution Tree.

Zemelman, S., Daniels, H., \& Hyde, A. (2012). Best practice: Today's standards for teaching and learning in America's schools. Portsmouth, $\mathrm{NH}$ : Heinemann.

Correspondence: Barbara Freeman, Consultant and Visiting Scholar, University of CaliforniaBerkeley, Graduate School of Education, Berkeley, California, United States of America 